\title{
Superconducting RF Cavities: Past, Present and Future
}

\author{
E. Chiaveri
}

\begin{abstract}
In the last two decades many laboratories around the world, notably Argonne (ANL), TJNAF (formerly CEBAF), CERN, DESY and KEK, undertook the development of the technology of superconducting (SC) accelerating cavities. The aim was either to increase the accelerator energy or to save electrical consumption or both. This technology has been used extensively in the operating machines showing good performances and strong reliability. At present, the technology using bulk niobium $(\mathrm{Nb})$ or $\mathrm{Nb}$ coated on copper $(\mathrm{Cu})$ is mature enough to be applied for many different applications, such as synchrotron light sources and spallation neutron drivers. Results, $R \& D$ work and future projects will be presented with emphasis on application to linear accelerators.
\end{abstract}

Index Terms-Author, please supply your own keywords or send a blank e-mail to keywords@ieee.org to receive a list of suggested keywords.

\section{INTRODUCTION}

I $\mathrm{N}$ THE last two decades laboratories around the world decided to develop the technology of superconducting accelerating cavities. The aims were to increase the accelerator energy and to save electricity consumption. Working at low temperatures with superconducting material makes it possible to attain high electromagnetic fields with low losses. At first the cavities were made of $\mathrm{Nb}$ sheet metal but, unfortunately, the accelerating fields which could be obtained were limited by quenches.

Since the level of power loss of a cavity is limited by the thermal conductivity of its wall, the efforts of the designers concentrated on improving the $\mathrm{Nb}$ purity, in order to increase this thermal conductivity at liquid helium temperature.

An alternative solution consists of replacing $\mathrm{Nb}$ with $\mathrm{Cu}$ as the material of cavity construction and depositing a thin $\mathrm{Nb}$ film $(\sim 1.2 \mu \mathrm{m})$ on the copper ( $\mathrm{Nb} / \mathrm{Cu}$ cavities). This approach offers inherent advantages: considerably higher stability against quenching, insensitivity to small magnetic fields and a higher quality factor than that of solid $\mathrm{Nb}$ at a given frequency and working temperature (4.2-4.5 K). It is evident that by replacing bulk $\mathrm{Nb}$ with an $\mathrm{Nb}$ layer, an important saving is achieved, even allowing for the additional cost of the more elaborate fabrication procedure. An interesting feature is the possibility of replacing $\mathrm{Nb}$ with a wider choice of superconducting materials [1].

\section{TeChNology DeVelopment}

In this paper two examples of the two technologies ( $\mathrm{Nb}$ bulk and $\mathrm{Nb} / \mathrm{Cu}$ ) will be presented, also taking into account the industrial production side.

\footnotetext{
Manuscript received August 5, 2002.

The author is with CERN, Geneva, Switzerland (e-mail: Enrico.Chiaveri@cern.ch).

Digital Object Identifier 10.1109/TASC.2003.812631
}

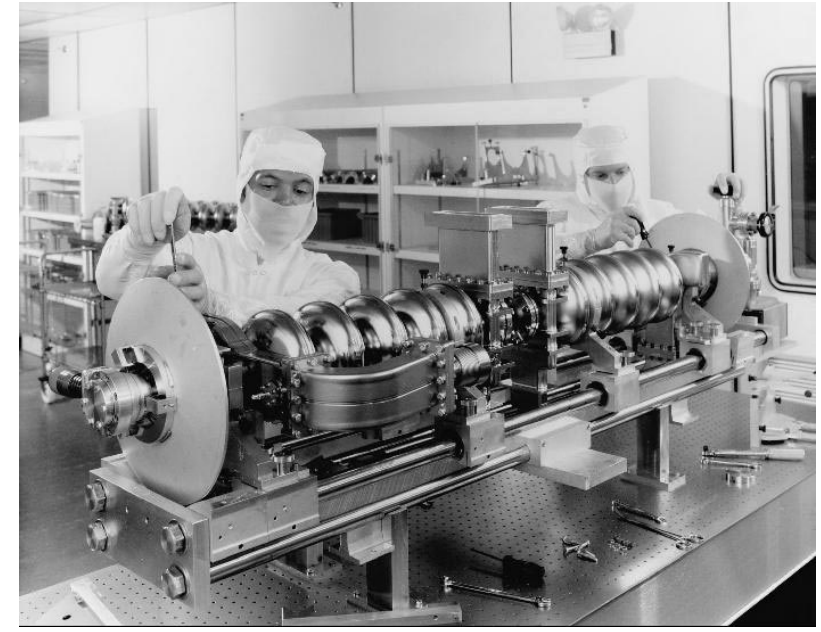

Fig. 1. CEBAF cavity assembly in the clean room.

\section{A. Nb Technology}

The CEBAF (Continuous Electron Beam Accelerator Facility) cavity design consists in total of about $40 \mathrm{Nb}$ parts which have to be manufactured, chemically treated and finally joined together by electron beam welding.

Concerning the main fabrication steps in industry, the first consists of making a visual inspection of the $\mathrm{Nb}$ sheets and choosing the side that will be exposed to the RF field. Half-cells are made using the deep drawing technique. All parts are degreased, soaked in $\mathrm{HCl}$ and $\mathrm{H}_{2} \mathrm{O}_{2}$ and the defects, if any, are removed using a special abrasive. Before electron beam (EB) welding the respective parts are chemically etched in an HF- $\mathrm{HNO}_{3}-\mathrm{H}_{3} \mathrm{PO}_{4}$ solution for a short time, rinsed in high-purity, filtered water, dried and packed under class 100 clean room conditions [2].

At CEBAF the complete cavities are chemically polished in a buffered chemical solution, the same as mentioned above, removing about $60 \mu \mathrm{m}$. Thorough rinsing in ultra-pure water, ultrasonic agitation and two-fold rinsing with reagent grade methanol follow. The RF windows, HOM couplers and vacuum valves are then mounted on the cavities in a class 100 clean room (Fig. 1).

\section{B. $\mathrm{Nb} / \mathrm{Cu}$ Technical Development}

In 1980 a development program aiming at the production of coated SRF cavities was started at CERN. The coating was achieved by sputtering using initially a bias diode configuration [1]. At the end of 1984 a series of 15 single-cell $500 \mathrm{MHz}$ cavities was produced, demonstrating that the accelerating fields and 


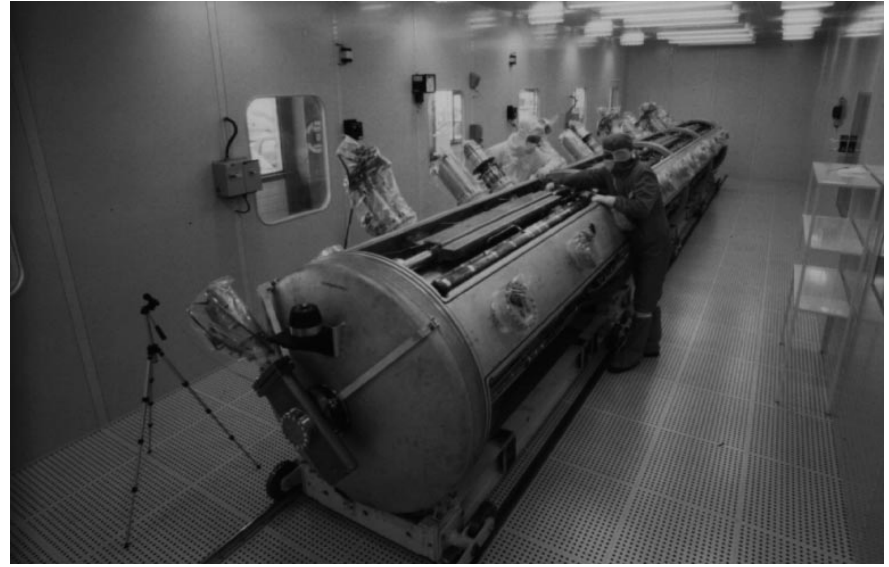

Fig. 2. LEP2 RF module assembly in the clean room.

$Q$ values are higher than those of bulk $\mathrm{Nb}$. A new electrode design was developed based on a cylindrical magnetron-sputtering configuration. This offered several advantages: increased sputtering rate, better adhesion and uniform thickness of the film. This technique was used to produce and install in the LEP tunnel a small series of Large Electron Positron collider (LEP) cavities (350 MHz) (12 cavities, three modules) in order to obtain information on long-term performance and to check reproducibility of performance. One of the major problems met in the research and development program was the substrate preparation before $\mathrm{Nb}$ coating. It turned out that in order to avoid "peel-off" of the $\mathrm{Nb}$ film any contamination must be avoided: in other words a cavity which has a total surface of about $6 \mathrm{~m}^{2}$ must be as clean as the silicon wafers of $20 \mathrm{~cm}^{2}$ surface used in the manufacture of VLSI (Very Large Scale Integration) integrated circuits. A special chemical treatment was developed at CERN for the whole cavity and the most critical phases of production, such as magnetron installation on the cavity, rinsing and drying, were carried out in a class 100 clean room.

\section{TECHNICAL DifFiculties ENCOUNTERED IN THE PRODUCTION}

Concerning the CEBAF production, almost all cavities are above the rather conservative specifications $\left(Q_{0}=2.4 \times 10^{9}\right.$ at $5 \mathrm{MV} / \mathrm{m}$ and $2 \mathrm{~K}$ ). They show consistently much higher fields during the vertical cavity pair testing, limited by either a thermal magnetic quench or electron loading. There is a wide spread in the quench fields observed, suggesting that improvements in the thermal stabilization of the $\mathrm{Nb}$ material would be needed if operation was desired at fields much higher than the specified value.

The surface preparation remains the critical operation in the fabrication process, even though for $\mathrm{Nb}$ cavities there is more experience available than for $\mathrm{Nb} / \mathrm{Cu}$ cavities.

The CERN acceptance specifications $\left(Q_{0}=3.4 \times 10^{9}\right.$ at 6 $\mathrm{MV} / \mathrm{m}$ and $4.5 \mathrm{~K}$ ) are fairly close to the best performance ever achieved in $\mathrm{Nb} / \mathrm{Cu}$ cavities at $352 \mathrm{MHz}$. Therefore a significant fraction of the cavity production has to be recovered by helium processing, rinsing and in difficult cases by replacement of the $\mathrm{Nb}$ layer. To help in cavity recovery, temperature mapping and visual inspection (video recording system) are applied systematically to all cavities not reaching the contractual performance at the first coating.

Observed defects are often the lack of coating adherence of the $\mathrm{Nb}$ film ("peel-off") or surface irregularities probably linked with the $\mathrm{Cu}$ substrate metallurgy and preparation [2]. A defect of $\mathrm{mm}^{2}$ size on the $6 \mathrm{~m}^{2}$ surface of a cavity can completely spoil the performance! It has been observed that most of the cases of "peel-off" of the $\mathrm{Nb}$ film are strongly related to staining of chemical products not having been correctly rinsed. A very careful control of procedures during chemical polishing is absolutely necessary, as well as a thorough maintenance of the chemistry installations. Additional cycles of electrolytical and chemical polishing of copper may be necessary.

Visual inspection of the cavity after chemical treatment (after grinding of a localized defect) was found to be a critical step in the production procedure. The fact that this inspection is by nature subjective requires very close collaboration with industry to transmit as rigorously and objectively as possible the relevant technical criteria. Quality assurance would require the fully objective definition of production steps for visual inspection and chemical treatment. The superconducting RF (SRF) cavity is an industrial reality (338 cavities for CEBAF, 288 cavities for CERN). The two techniques ( $\mathrm{Nb}$ and $\mathrm{Nb} / \mathrm{Cu}$ ) are very well managed by industry from $\mathrm{Nb}$ sheet or $\mathrm{Cu}$ sheet to the final product within specifications base on RF performance at liquid helium temperature [2].

\section{Performance AND Reliability of LEP CAVITIES}

One of the most important issues in SRF cavity development is the level of reliability of these components in the accelerator environment. Here the LEP2 experience will be reported on as an example of a large number of SRF cavities (288) operating on an accelerator for five years.

As in any RF system, the SC cavities must be reconditioned after a long interruption. This is the case in particular at the end of the annual long machine shutdown. One or two weeks are dedicated to cavity conditioning at the start of a new run.

Cavity conditioning can be done by simple RF processing, i.e., a gradual increase of the RF field, limited by the vacuum level in the cavity and RF coupler. To reach the highest fields it is often necessary to use pulse processing (a few ms-long RF pulses at $100 \mathrm{~ms}$ intervals). A good indication of the quality of the cavity is the level of radiation produced by the spurious electrons (from the emitters, dust particles) accelerated all along a module. Typical levels range from a few $\mathrm{krad} / \mathrm{h}$ to $50 \mathrm{krad} / \mathrm{h}$. In the most difficult cases, even in the LEP tunnel, helium processing was used. With all these techniques the available accelerating field in LEP was significantly above its design value of $6 \mathrm{MV} / \mathrm{m}$.

Cryogenic measurements of the cavity quality factor $Q_{0}$ (the only measurements of $Q_{0}$ available when the cavities are installed) do not show any sign of degradation of the quality of the $\mathrm{Nb}$ layer. There was only one incident that required removal of a module out of the tunnel. This module was accidentally exposed to a fast flow of clean nitrogen, following a human error in controlling the vacuum valves. Normally the cavities are vented 
to atmospheric pressure with a very slow flow of nitrogen to avoid transport of dust particles. The module was removed from the beam line (two-day operation) and completely disassembled. Each cavity was rinsed separately with ultra-pure water and subsequently RF tested ( $Q_{0}$ vs. $E$ curve) to check again its performance. The module was then reassembled and reinstalled in LEP. (The total duration of this repair was about one month [3].)

\section{Present and Future Projects}

Many projects in the future are going to use SRF technology, e.g., synchrotron light projects (Diamond, Soleil, Taiwan and Canadian light sources), the heavy ion injector PIAVE [4] and the U.S. rare-isotope accelerator facility [5]. In this paper The most important of the present and future projects related to linear accelerators will be presented.

\section{A. Spallation Neutron Source (SNS)}

The SNS is an accelerator-based pulsed neutron source, which provides thermal neutrons to condensed matter research. The baseline accelerator system is to deliver a beam power greater than 1.4 MW and consists of an $\mathrm{H}$ ion source, a radio-frequency quadrupole (RFQ), a $1 \mathrm{GeV}$ linac, a compressor ring and associated beam transport systems. The time-averaged beam current is $1.4 \mathrm{~mA}$.

In January 2000, the main section of the SNS linac was changed from normal conducting copper technology to a superconducting technology after extensive reviews. The new linac baseline configuration consists of a drift tube linac (DTL), which accelerates an incoming $2.5 \mathrm{MeV}$ beam from an RFQ to $70 \mathrm{MeV}$, a coupled cavity linac (CCL), which accelerates the 70 $\mathrm{MeV}$ beam to $186 \mathrm{MeV}$, and an SC section, which accelerates the $186 \mathrm{MeV}$ beam to $1 \mathrm{GeV}$. The superconducting portion consists of two parts, a medium- $\beta$ and a high- $\beta$ section. In spite of the fact that there have been many design studies for high-power proton SC linacs during the past decade, the SNS linac will be the first high-intensity proton SC linac [6].

1) Status of $R \& D$ and Construction: The schedule for the SNS SRF linac consists of a two-year prototyping R\&D period and a two-year period of cavity construction along with design/construction of the cryogenic system and transfer lines. Some of the key prototyping items include four medium- $\beta$ and two high- $\beta$ cavities. Fig. 3 shows the SNS prototype cavities: $\beta=0.61$ and 0.81 . As for electromagnetic performance based on the vertical tests without titanium He vessels, both the medium- and high- $\beta$ cavities exceeded the design goals after proper treatments.

\section{B. Superconducting $\mathrm{H}^{-}$Linac (SPL)}

The ever-increasing flux of secondary particles requested by physics experiments can only be met using higher power proton beams. These requests have re-activated the study of a Superconducting Proton Linac (SPL) already proposed as an upgraded injector for the CERN PS. Triggered by a previous proposal to re-use the LEP-RF hardware for the proton driver of an energy amplifier [7], this machine was originally intended for accelerating mainly protons.

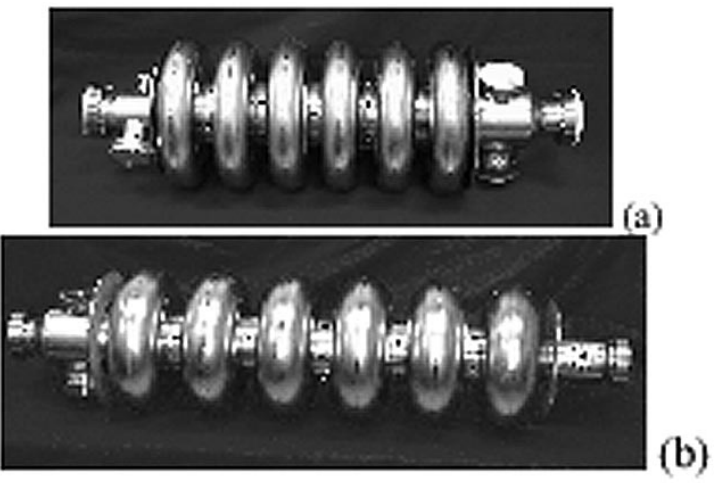

Fig. 3. SNS prototype cavities: (a) $\beta=0.61$ and (b) $\beta=0.81$

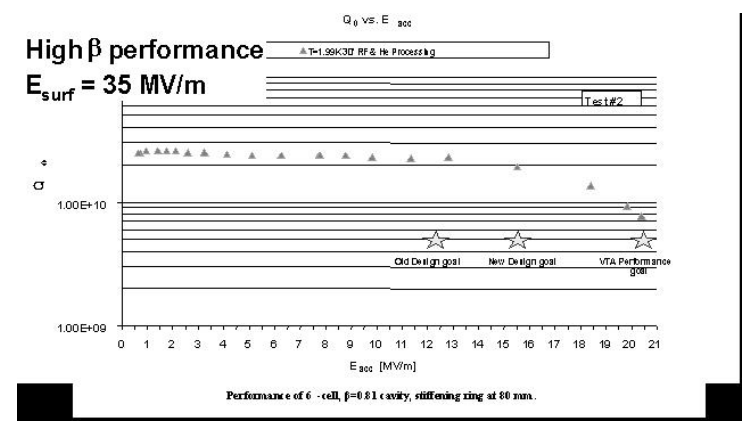

Fig. 4. Performance of 6-cell $(\beta=0.81)$ cavity.

The following studies show the advantages of a common $\mathrm{H}^{-}$ operation for all users. The linac parameters must be defined to suit the following possible uses:

- PS ring for the LHC and other high-intensity fixed-target applications

- Increased flux $(\sim \times 2)$ for CERN Neutrinos to Gran Sasso (CNGS)

- Increased flux for Anti-proton Decelerator

- Increased flux for Neutron Time of Flight (TOF) experiments

- ISOLDE: increased flux, higher duty cycle, multiple energies

- Driver for a future neutrino factory

- Generation of a conventional neutrino beam for mediumdistance experiments $(\sim 100 \mathrm{~km})$

The present design makes extensive use of the large inventory of RF equipment dismantled from LEP. The $800 \mathrm{~m}$ long SC linac that accelerates the $\mathrm{H}^{-}$ions to $2.2 \mathrm{GeV}$ re-uses all klystrons and $60 \%$ of the LEP modules in its high-energy part (see Fig. 5). New $\beta=0.52$ and $\beta=0.7$ acceleration modules are assumed between 120 and $390 \mathrm{MeV}$. Between $390 \mathrm{MeV}$ and $1 \mathrm{GeV}$, LEP cryostats are re-used, equipped with new 5-cell, $\beta=0.8$ cavities.

Below $120 \mathrm{MeV}$, room-temperature accelerating structures are employed. Leaving the ion source at $45 \mathrm{keV}$, the $\mathrm{H}^{-}$beam is bunched at $352 \mathrm{MHz}$ and accelerated to $3 \mathrm{MeV}$ in an RFQ. It then passes through a transfer line equipped with fast deflecting electrostatic kickers ("choppers") which eliminate the unwanted bunches onto a collector and provides the proper time structure for an optimum longitudinal capture in the accumulator. Further acceleration to $120 \mathrm{MeV}$ is made cascading an RFQ, a 


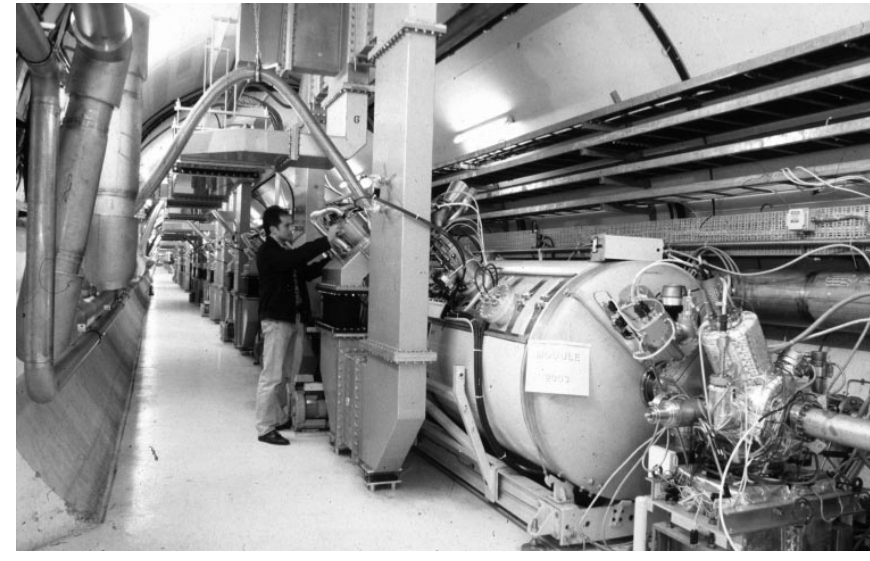

Fig. 5. LEP2 RF module in the tunnel.

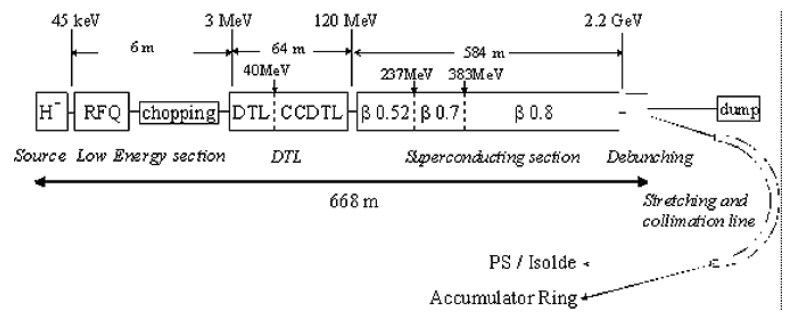

Fig. 6. SPL block diagram.

Drift Tube Linac (DTL) and a Cavity Coupled Drift Tube Linac (CCDTL) [8].

\section{Advanced Accelerator Applications (AAA) Project}

The Advanced Accelerator Applications Program goal is to develop technologies required for transmuting radioactive waste into shorter lived, less toxic material. As part of this program, an accelerator provides the required hard neutron spectrum through a spallation process. The proton beam must have a high reliability at continuous currents levels up to $13 \mathrm{~mA}$. The accelerator front end is based on LEDA (Los Alamos Low Energy Demonstration Accelerator), a $75 \mathrm{KeV}$ injector followed by a 6.7 MeV RFQ. Because of the low-current and high-reliability requirement, the remaining accelerator structure is superconducting. From $6.7 \mathrm{MeV}$ to $109 \mathrm{MeV}$, the accelerator uses spoke resonators with $\beta \mathrm{s}$ of 0.175 and 0.34 . From $109 \mathrm{MeV}$ to the final energy of $600 \mathrm{MeV}$, the accelerator uses elliptical cavities with $\beta$ s of 0.48 and 0.64 .

1) AAA Linac Requirements: The AAA program requires a hard neutron spectrum that can be produced by a spallation process from a proton beam. The proton beam for the ADTF (Accelerator Demonstration Test Facility) linac must have highreliability at continuous-current levels up to $13 \mathrm{~mA}$. The power of the ADTF linac is determined by the design of the subcritical multiplier. With a reactor equivalent power of $100 \mathrm{MW}$ and with the multiplier keff in a range of 0.92 to 0.98 , the maximum proton beam power is $8 \mathrm{MW}$ (Fig. 7).

2) AAA Linac Design: An SCRF linac has been chosen for the ADTF linac because, compared to linacs using traditional room-temperature (RT) copper technology, SCRF linacs are more power efficient and expected to have higher reliability. A comparison of SRF and RT technologies has been reviewed

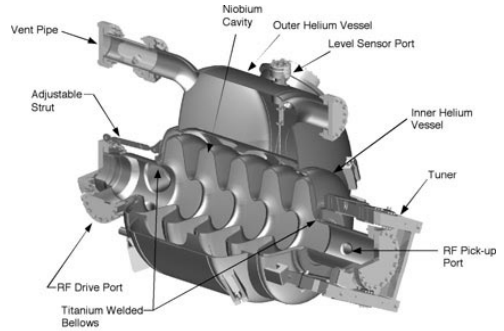

Fig. 7. 5-cell $(\beta=0.8)$ design.

recently at Los Alamos by a panel of accelerator experts. An SC linac significantly lowers the total linac $\mathrm{AC}$ power requirement. For a $600-\mathrm{MeV}$ linac at $13 \mathrm{~mA}$, an SRF linac needs $23 \mathrm{MW}$ of $\mathrm{AC}$ power as compared to $80 \mathrm{MW}$ needed for an RT (room temperature) linac [9].

\section{Tera Electron Volt Energy Superconducting Linear Collider (TESLA)}

There is a worldwide consensus that the next large project for high-energy physics must be a high luminosity $\mathrm{e}^{+} \mathrm{e}^{-}$linear collider of about $500 \mathrm{GeV} \mathrm{CM}$ energy, upgradeable to higher energies of order $1 \mathrm{TeV}$.

A major challenge for all linear collider concepts is to obtain a large collision rate (luminosity) of electrons and positrons at the interaction point. This requires very small spot sizes of the beams at the collision point and high beam powers.

The TESLA approach differs from the other designs by the choice of SC accelerating structures as its basic technology. The TESLA linear collider based on SC accelerating structures is ideally suited to meet the requirements needed for a large collision rate, namely very small beam sizes and high beam power.

Superconducting technology provides important advantages for a linear collider. As the power dissipation in the cavity walls is extremely small, the power transfer efficiency from the RF source to the beam is very high, thus keeping the electrical power consumption within acceptable limits $(\sim 100 \mathrm{MW})$, even for a high average beam power. The high beam power is the first essential requirement for obtaining a high rate of electronpositron collisions.

The second requirement is extremely small sizes of the electron and positron beam at the interaction point. The relatively low RF frequency of the TESLA linear accelerators is ideally suited for conserving the ultra-small size of the beams during acceleration. When a beam is accelerated in a linear accelerator, the charged particles induce electromagnetic fields (so-called wake fields), which act back on the beam itself and can spoil its quality by increasing the energy spread and the beam size. As these wake-field effects decrease strongly with increasing distance between the beam and the surrounding cavity walls, wake fields are much weaker in the larger cavities of accelerators working at low RF frequencies than in smaller cavities operating at higher frequencies.

The advantage of SC technology has been acknowledged from the very beginning of the research and development on linear colliders, but the technology was considered to be considerably more expensive than conventional technologies. 
By a focused development program, started in 1992, the international TESLA collaboration in co-operation with industry succeeded in developing SC cavities which are capable of generating an accelerating gradient five times larger than before 1990. In addition a reduction of the cost per meter of accelerator by a factor of four was achieved. Together, these achievements provide the basis for a realistic SC linear collider with all its advantages [8].

1) The Baseline Design: The test linac of TTF (TESLA Test Facility [9]), set up as a complete system test comprising prototypes of all subsystems necessary for the TESLA linear accelerator, has been operated successfully for several years. Therefore the technical layout of the TESLA linear accelerators has a solid basis. The cryogenic accelerator modules for TESLA will be about $17 \mathrm{~m}$ long, containing twelve 9-cell cavities instead of 8 for the $12 \mathrm{~m}$ long TTF modules. This way a cost reduction and a higher filling factor of the linear accelerator are achieved. For the fixed length of the linear collider of about $33 \mathrm{~km}$ a gradient of $23.4 \mathrm{MV} / \mathrm{m}$ is needed for $500 \mathrm{GeV}$ operations. The total number of cavities for the main linacs is 21024 corresponding to 1752 modules and 584 RF stations.

Higher beam energies than for the baseline design are possible within the site length since the physical limit for the gradient in niobium structures at $2 \mathrm{~K}$ is above $50 \mathrm{MV} / \mathrm{m}$ and several 9-cell cavities have already reached gradients above $30 \mathrm{MV} / \mathrm{m}$ at TTF.

Electro-polishing followed by low-temperature bake-out-a technology being developed by KEK - has yielded systematically higher performance single-cell cavities, with gradients up to $42 \mathrm{MV} / \mathrm{m}$.

Based on the ongoing progress in building high gradient cavities we assume that TESLA will be built from the very beginning with superstructures and mostly with cavities reaching gradients of on average $35 \mathrm{MV} / \mathrm{m}$, thus allowing a total collision energy of $800 \mathrm{GeV}$ without major changes to the accelerator modules. The beam delivery system and the magnets in the linear accelerators have been designed for a beam energy of up to $400 \mathrm{GeV}$.

Collision energies above $500 \mathrm{GeV}$ can be reached in two steps: A total energy of $650 \mathrm{GeV}$ can already be obtained in the baseline design as the cooling plant capacity has a 50\% overhead, thus allowing the gradient in the cavities to be increased by $20-30 \%$.

In order to reach $800 \mathrm{GeV}$ at maximum luminosity two upgrades are required: the cooling capacity of the cryogenic plant must be increased and the number of the RF stations must be doubled.

\section{E. Neutrino Factory}

The neutrino factory requires nearly $500 \mathrm{~m}$ of $200 \mathrm{MHz}$ SRF cavities to provide $7.5 \mathrm{GV}$. Such a facility is more demanding than the LEP where $500 \mathrm{~m}$ of $\mathrm{Nb}$-coated $\mathrm{Cu}$ cavities provide 3 $\mathrm{GV}$ of acceleration.

Acceleration of a muon beam is challenging because of the large phase space and short muon lifetime. To minimize muon loss from decay, the highest possible gradient is necessary. SRF offers gradients of $15 \mathrm{MV} / \mathrm{m}$ and reduces the peak RF power needed by virtue of long till times made affordable by superconductivity. Superconducting RF cavities also provide a large

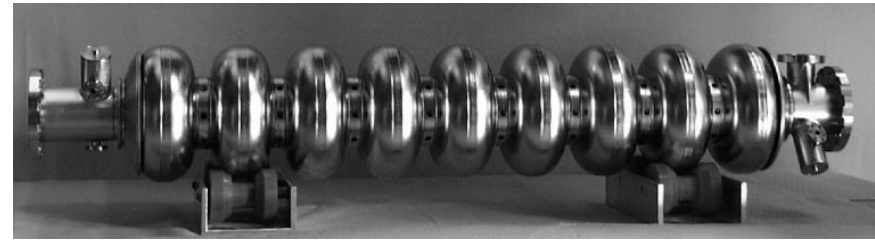

Fig. 8. TESLA cavity.

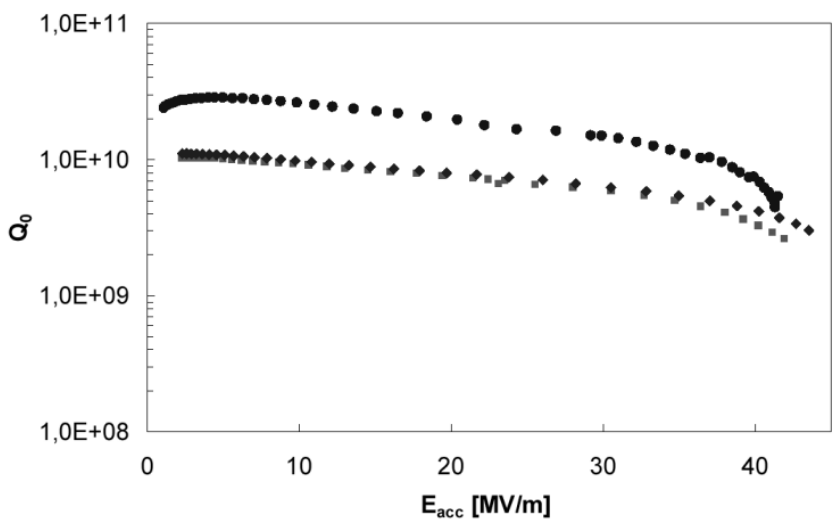

Fig. 9. Performance of 9-cell cavity.

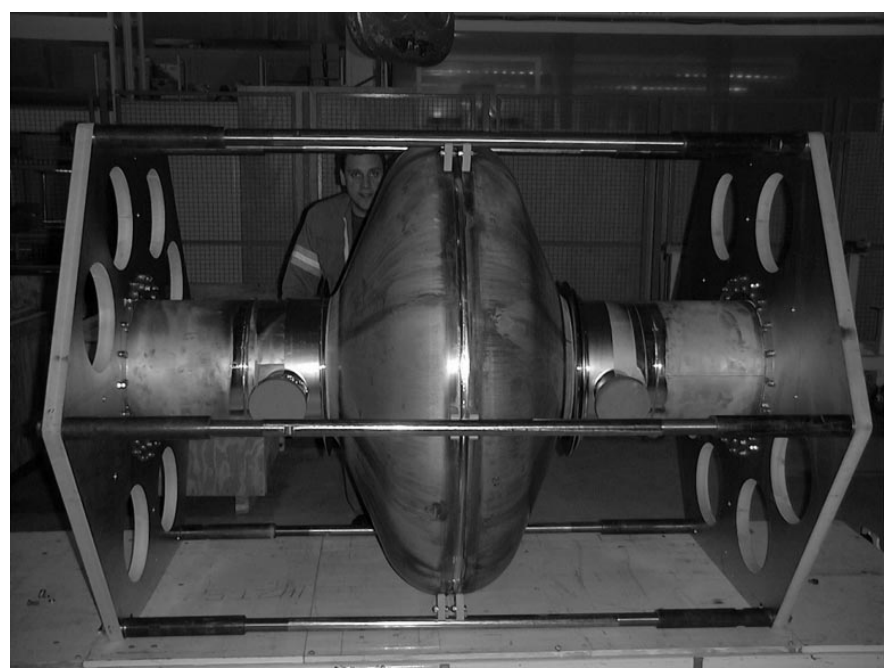

Fig. 10. $200 \mathrm{MHz} \mathrm{Nb/Cu}$ single cell cavity.

aperture that preserves beam quality and beam stability. The acceleration system starts with a linac from about $500 \mathrm{MeV}$ to 2.5 $\mathrm{GeV}$ followed by a four-pass recirculating linac to the final energy of $20 \mathrm{GeV}$. The need for very large beam acceptances drives the design to a low RF frequency of $200 \mathrm{MHz}$. Under an agreement between CERN and Cornell University such a cavity has been built and $\mathrm{Nb}$ coated at CERN. At present the single-cell cavity is under cryogenic test at Cornell University [12].

\section{CONCLUSION AND FUTURE}

It should be stressed that $\mathrm{SRF} \mathrm{Nb}$ and $\mathrm{Nb} / \mathrm{Cu}$ technologies have demonstrated their performance on the largest scale and they are very competitive for a number of applications.

In future the SNS, TESLA, SPL, AAA and other projects will require mass production of SRF cavities. It will become more and more important to find correlations between the fabrication 
steps and the cavity performance to avoid a large scatter in the final results or the need of expensive recovery procedures. The rigorous control of the surface quality is certainly the key to an acceptable success rate for cavity production. The companies should be involved from the very beginning of the project. They are best experienced to propose the simplest and most economic fabrication procedures, especially for all mechanical constructions.

Finally I would like to stress that, whatever quality control we can apply, highly motivated, well-trained, fully committed, well-managed technical staff remain the key for the success of a project.

\section{ACKNOWLEDGMENT}

The author is grateful to R. Losito (CERN), H. Padamsee (Cornell University), L. Lilje (DESY), N. Holtkamp (SNS), R. Parodi (INFN-Genova), A. Facco (INFN-Legnaro) and P. Kneisel (TJNAF) for helping me, with information and pictures, to prepare this conference. I wish to thank also Tricia Martucci for preparation and critical reading of the manuscript.

It is a pleasure to acknowledge all the people who with their ability, competence, dedication and long-lasting commitment have made possible the excellent results obtained by these programs.

\section{REFERENCES}

[1] E. Chiaveri, "Large-scale industrial production of superconducting cavities," in 7th Workshop on RF Superconductivity, Gif-sur-Yvette, France, Oct. 17-20, 1995.

[2] — , "Production by industry of a large number of superconducting cavities: Status and future," Particle Accelerators, 1996.

[3] D. Boussard and E. Chiaveri, "The LEP superconducting RF system: Characteristics and operational experience," in Utilization and Reliability of High Power Proton Accelerators, Mito, Japan, October 13-15, 1998.

[4] A. Facco et al., "Superconducting RF activities at LNL," in 10th Workshop on RF Superconductivity, Tsukuba, Japan, September 6-11, 2001.

[5] K. W. Shepard, "The U.S. RIA Project SRF Linac,".

[6] Y. Cho for the SNS Collaboration, "Evolution of the SNS superconducting proton linac," in 10th Workshop on RF Superconductivity, Tsukuba, Japan, September 6-11, 2001.

[7] C. Rubbia and J. A. Rubio, "A tentative programme toward a full scale energy amplifier," CERN/LHC/96-11.

[8] E. Chiaveri, "A high-power superconducting $\mathrm{H}^{-}$linac (SPL) at CERN," in 10th Workshop on RF Superconductivity, Tsukuba, Japan, September 6-11, 2001.

[9] R. L. Sheffield, "Superconducting technologies for the advanced accelerator application program," in 10th Workshop on RF Superconductivity, Tsukuba, Japan, September 6-11, 2001.

[10] D. Trines, "TESLA," in 10th Workshop on RF Superconductivity, Tsukuba, Japan, September 6-11, 2001.

[11] A. Matheisen, "Status of the RF superconductivity activities at DESY," in 10th Workshop on RF Superconductivity, Tsukuba, Japan, September 6-11, 2001.

[12] H. Padamsee, "SRF for neutrino factories," in 10th Workshop on $R F$ Superconductivity, Tsukuba, Japan, September 6-11, 2001. 